\title{
The Chromosome 9p21 Variant Not Predicting Long-Term Cardiovascular Mortality in Chinese with Established Coronary Artery Disease: An Eleven-Year Follow-Up Study
}

\author{
I-Te Lee, ${ }^{1,2,3}$ Mark O. Goodarzi, ${ }^{4,5}$ Wen-Jane Lee, ${ }^{6}$ Jerome I. Rotter, ${ }^{5}$ Yii-der Ida Chen, ${ }^{5,7}$ \\ Kae-Woei Liang, ${ }^{8}$ Wen-Lieng Lee, ${ }^{8}$ and Wayne H.-H. Sheu ${ }^{1,2,3}$ \\ ${ }^{1}$ Division of Endocrinology and Metabolism, Department of Internal Medicine, Taichung Veterans General Hospital, \\ Taichung 40705, Taiwan \\ ${ }^{2}$ School of Medicine, Chung Shan Medical University, Taichung 40201, Taiwan \\ ${ }^{3}$ School of Medicine, National Yang-Ming University, Taipei 11221, Taiwan \\ ${ }^{4}$ Division of Endocrinology, Diabetes and Metabolism, Cedars-Sinai Medical Center, Los Angeles, CA 90048, USA \\ ${ }^{5}$ Medical Genetics Institute, Cedars-Sinai Medical Center, Los Angeles, CA 90048, USA \\ ${ }^{6}$ Department of Medical Research, Taichung Veterans General Hospital, Taichung 40705, Taiwan \\ ${ }^{7}$ Department of Ob/Gyn, Cedars-Sinai Medical Center, Los Angeles, CA 90048, USA \\ ${ }^{8}$ Cardiovascular Center, Taichung Veterans General Hospital, Taichung 40705, Taiwan
}

Correspondence should be addressed to Wayne H.-H. Sheu; whhsheu@vghtc.gov.tw

Received 30 November 2013; Revised 17 February 2014; Accepted 19 February 2014; Published 2 April 2014

Academic Editor: Amelie Bonnefond

Copyright (C) 2014 I-Te Lee et al. This is an open access article distributed under the Creative Commons Attribution License, which permits unrestricted use, distribution, and reproduction in any medium, provided the original work is properly cited.

\begin{abstract}
Introduction. We examined whether the variant at chromosome 9p21, rs4977574, was associated with long-term cardiovascular mortality in Han Chinese patients with coronary artery disease (CAD). Methodology. Subjects who underwent coronary angiography for chest pain were consecutively enrolled. Fasting blood samples were collected for laboratory and genotype assessments. The information was correlated with data collected from the national death database. Results. There were 925 cases with CAD and 634 without CAD enrolled in the present study. The G allele conferred a significant increase in risk of CAD (odds ratio $=1.47, P=0.003$ in the dominant model; odds ratio $=1.36, P=0.018$ in the recessive model). During a median of 11 years (inter-quartile range between 5.2 and 12.5 years) of follow-up, neither the total nor the cardiovascular mortality was different among CAD subjects with different genotypes. Using Cox regression analysis, genotypes of rs 4977574 still failed to predict cardiovascular mortality (hazard ratio $=1.25, P=0.138$ in the dominant model; hazard ratio $=1.05, P=0.729$ in the recessive model). Conclusions. The rs4977574 at chromosome 9p21 is associated with presence of CAD in Han Chinese. However, rs4977574 could not predict cardiovascular mortality in these CAD subjects during the eleven-year period of the study.
\end{abstract}

\section{Introduction}

Coronary artery disease (CAD) is the leading cause of death worldwide [1]. Heredity plays an important role in CAD susceptibility [2]. The chromosome 9p21 region has been shown to be associated with CAD, and several single nucleotide polymorphisms (SNPs) selected from Caucasians have been replicated in Chinese [3-5]. However, SNPs in chromosome 9p21 contribute diverse intensities of risk to cardiovascular disease, and it has been reported that the effect size of identified SNPs is dependent on the chosen population [6-8].

Among the tag SNPs, rs4977574 has been initially demonstrated to be associated with risk of CAD in the Myocardial Infarction Genetics Consortium (MIGen) study [9]. The association between rs4977574 and CAD has also been found in several populations [10-13]. However, a small study comprising 334 subjects did not show any significant association between CAD risk and rs4977574 in a Chinese population [14]. 
Although rs4977574 was recently reported to be associated with cardiovascular incidence in the subjects without CAD at baseline [15], results from longitudinal assessments between 9p21 variants and cardiovascular mortality in subjects with established CAD were inconsistent. In the Global Registry of Acute Coronary Events (GRACE) genetic study, the 9p21 variant showed a significant association with cardiovascular death [16]. However, Virani et al. [17] recently reported that the $9 \mathrm{p} 21$ variant was not associated with cardiovascular outcome. In order to clarify the longterm impact of 9p21 variant on cardiovascular mortality in CAD patients, we therefore examined the polymorphism of rs4977574 in a Taiwanese cohort of Han Chinese descent in an eleven-year follow-up duration.

\section{Material and Methods}

2.1. Patients. The participants were enrolled during hospitalization in the Cardiovascular Department in Taichung Veterans General Hospital between March 1999 and March 2000. The study comprised both case-control and follow-up assessments for subjects undergoing coronary angiography due to angina and clinical suspicion of ischemic heart disease. Blood samples were collected after the subjects had fasted overnight. Mortality data up to 2011 were collected for the follow-up assessment. The study protocol was approved by the Institutional Review Board of Taichung Veterans General Hospital, Taichung, Taiwan.

2.2. Methods. Genomic DNA was extracted from patients' peripheral blood leukocytes using a QIAamp DNA Blood Mini Kit (Qiagen, Hilden, Germany). Genotypes of rs4977574 were determined using TaqMan allelic discrimination assay (Custom TaqMan SNP Genotyping Assay). The genotyping reaction was amplified and detected on a StepOnePlus Real-Time PCR (Applied Biosystems, Foster City, USA).

Plasma glucose was determined using the glucose oxidase-peroxidase method (Wako Diagnostics, Tokyo, Japan). Serum cholesterol and triglyceride levels were determined by the enzymatic method using commercial kits (Wako Diagnostics, Tokyo, Japan). High-density lipoprotein (HDL) cholesterol was determined after the precipitation of apo B-containing lipoproteins. Low-density lipoprotein (LDL) cholesterol concentration was calculated using the formula of Friedewald et al. [18] if fasting serum triglyceride was less than $4.5 \mathrm{mmol} / \mathrm{L}$. Otherwise, LDL cholesterol was determined after separation of very-low-density lipoprotein (VLDL) from serum by ultracentrifugation and precipitation of apo B-containing particles.

2.3. Exposure Information. The subjects were assigned to the CAD group if they fulfilled one or both of the following criteria: (1) a history of myocardial infarction or coronary revascularization or (2) $50 \%$ or more luminal narrowing in any coronary artery according to angiography. Subjects who did not fulfill any of these criteria were assigned to the nonCAD group. Diabetes mellitus was defined as (1) a history of diabetes mellitus or use of antidiabetic medications or (2) fasting blood glucose levels equal to or higher than $7.0 \mathrm{mmol} / \mathrm{L}$. Hypertension was defined as (1) a history of hypertension or use of antihypertensive medications or (2) systolic blood pressure higher than $140 \mathrm{mmHg}$ or diastolic blood pressure higher than $90 \mathrm{mmHg}$.

The coronary artery lesions were assessed at the angiography viewing workstation using software for quantitative analysis (Philips Inturis Suite, R2.2, Philips Medical Systems, Eindhoven, Netherlands). The baseline CAD severity was evaluated by Gensini score, which was calculated by the sum of obstructive severity score times the lesion location score system [19].

The mortality data were provided by the Collaboration Center of Health Information Application (CCHIA), Department of Health, Executive Yuan, Taiwan. The causes of death were determined according to the International Classification of Disease, 9th Revision, Clinical Modification (ICD-9 CM) diagnostic criteria. Cardiovascular disease was defined as CAD (402, 404, 410, 411, 412, 413, 414, 426, 427, 428, and 429), cerebrovascular disease $(430,431,432,433,434,435,436,437$, and 785.9), and peripheral artery disease (440.2, 443.8, 443.9, and 444.2) [20].

2.4. Statistical Analysis. Data are presented as mean \pm standard deviation (SD). The genotype distribution was tested for Hardy-Weinberg equilibrium by the goodness-of-fit test. Chi-square test was used to assess the differences in gender, genotypes, diabetes, hypertension, and smoking status. The differences in continuous variables at baseline were analyzed by independent $t$-test. Multivariate logistic regression analyses were used to assess the genetic effects on CAD at baseline. Multivariable Cox proportional-hazard regression analyses were used to examine the cardiovascular mortality between genotypes. Statistical analysis was performed using SPSS 19.0 (SPSS Inc., Chicago, IL, USA).

\section{Results}

3.1. Case-Control Assessments at Baseline. A total of 1559 subjects were enrolled with 925 in the CAD group and 634 in the non-CAD group (Figure 1). The distribution of alleles was in agreement with the Hardy-Weinberg equilibrium. The clinical features of the two study groups are shown in Table 1. The CAD group was significantly older with male dominance compared with the non-CAD group (both $P$ values less than $0.001)$. There were significantly higher proportions of current smoking status $(P<0.001)$, hypertension $(P=0.032)$, and diabetes mellitus $(P<0.001)$ in the CAD group than those in the non-CAD group. Fasting serum concentrations of LDL cholesterol and total cholesterol were also significantly higher in the CAD group than those in the non-CAD group (both $P$ values less than 0.001$)$. The genotype distributions showed that $G$ allele of rs4977574 was overrepresented in the CAD group compared to the non-CAD group $(P=0.002$ for genotype distribution and $P=0.001$ for allele distribution, resp.). The risk allele remained independently associated with CAD after adjusting for age, gender, smoking, hypertension, 


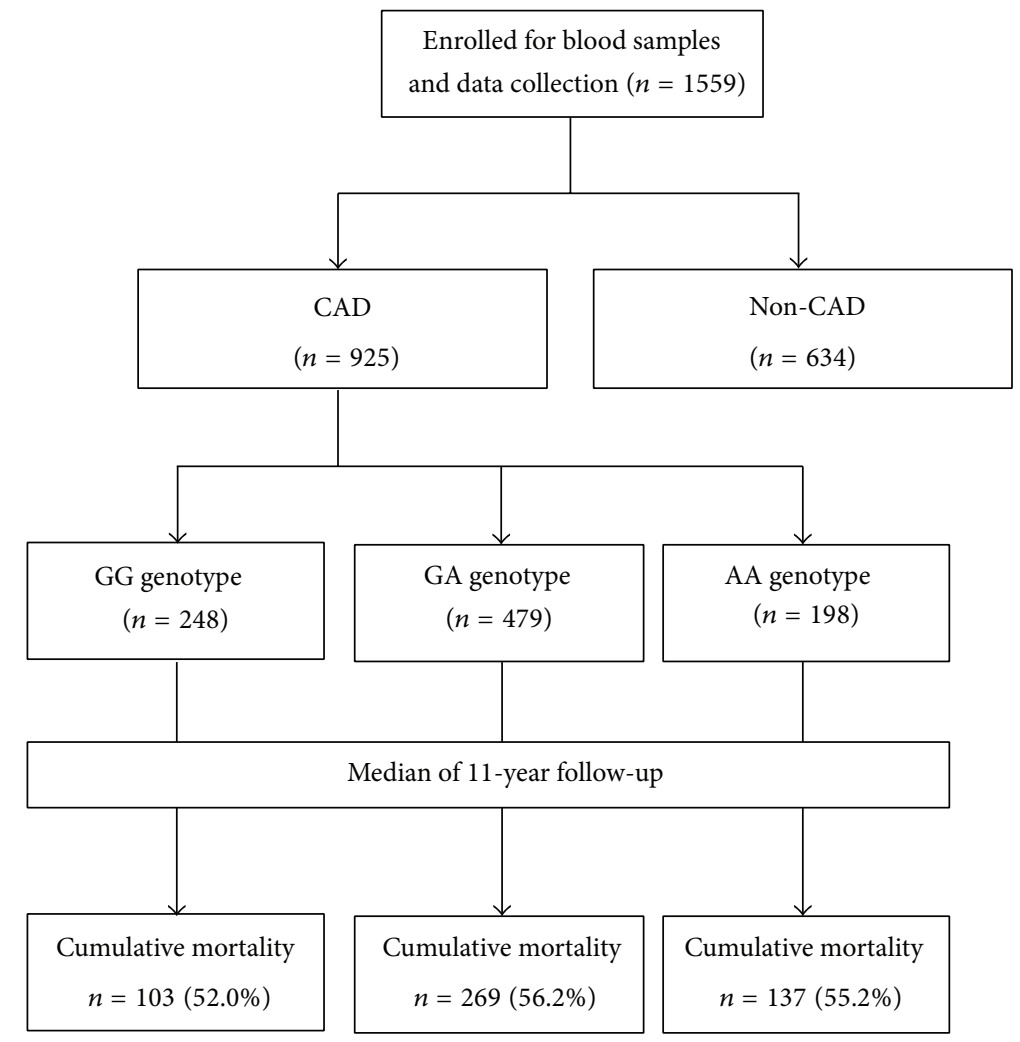

FIGURE 1: Flow diagram of enrollment of study subjects.

TABLE 1: The clinical characteristics and genotypic frequency of study subjects at baseline.

\begin{tabular}{|c|c|c|c|}
\hline & $\mathrm{CAD}(N=925)$ & Non-CAD $(N=634)$ & $P$ \\
\hline Age (year) & $67.6 \pm 10.0$ & $64.5 \pm 10.8$ & $<0.001$ \\
\hline Male, $n(\%)$ & $742(80.2 \%)$ & $408(64.4 \%)$ & $<0.001$ \\
\hline BMI $\left(\mathrm{kg} / \mathrm{m}^{2}\right)$ & $25.3 \pm 3.3$ & $25.3 \pm 3.5$ & 0.954 \\
\hline Systolic BP (mmHg) & $126 \pm 13$ & $126 \pm 11$ & 0.490 \\
\hline Diastolic BP (mmHg) & $71 \pm 10$ & $71 \pm 11$ & 0.813 \\
\hline Current smoking, $n(\%)$ & $260(28.1 \%)$ & $128(20.2 \%)$ & $<0.001$ \\
\hline Hypertension, $n(\%)$ & $466(50.4 \%)$ & $280(44.2 \%)$ & 0.032 \\
\hline Diabetes mellitus, $n(\%)$ & $344(37.2 \%)$ & $137(21.6 \%)$ & $<0.001$ \\
\hline Triglyceride (mmol/L) & $1.6 \pm 1.3$ & $1.5 \pm 1.4$ & 0.045 \\
\hline Total cholesterol (mmol/L) & $4.8 \pm 1.2$ & $4.4 \pm 1.2$ & $<0.001$ \\
\hline HDL cholesterol (mmol/L) & $1.1 \pm 0.3$ & $1.2 \pm 0.4$ & 0.312 \\
\hline LDL cholesterol (mmol/L) & $2.9 \pm 1.0$ & $2.6 \pm 0.9$ & $<0.001$ \\
\hline Fasting glucose (mmol/L) & $6.8 \pm 2.7$ & $6.1 \pm 2.1$ & $<0.001$ \\
\hline Genotype (rs4977574) & & & 0.002 \\
\hline AA & $198(21.4 \%)$ & $181(28.5 \%)$ & \\
\hline AG & $479(51.8 \%)$ & $318(50.2 \%)$ & \\
\hline GG & $248(26.8 \%)$ & $135(21.3 \%)$ & \\
\hline Allele (rs4977574) & & & $P=0.001$ \\
\hline A & $875(47.3 \%)$ & $680(53.6 \%)$ & \\
\hline G & $975(52.7 \%)$ & $588(46.4 \%)$ & \\
\hline
\end{tabular}

BMI: body mass index, BP: blood pressure, HDL: high-density lipoprotein, and LDL: low-density lipoprotein. 


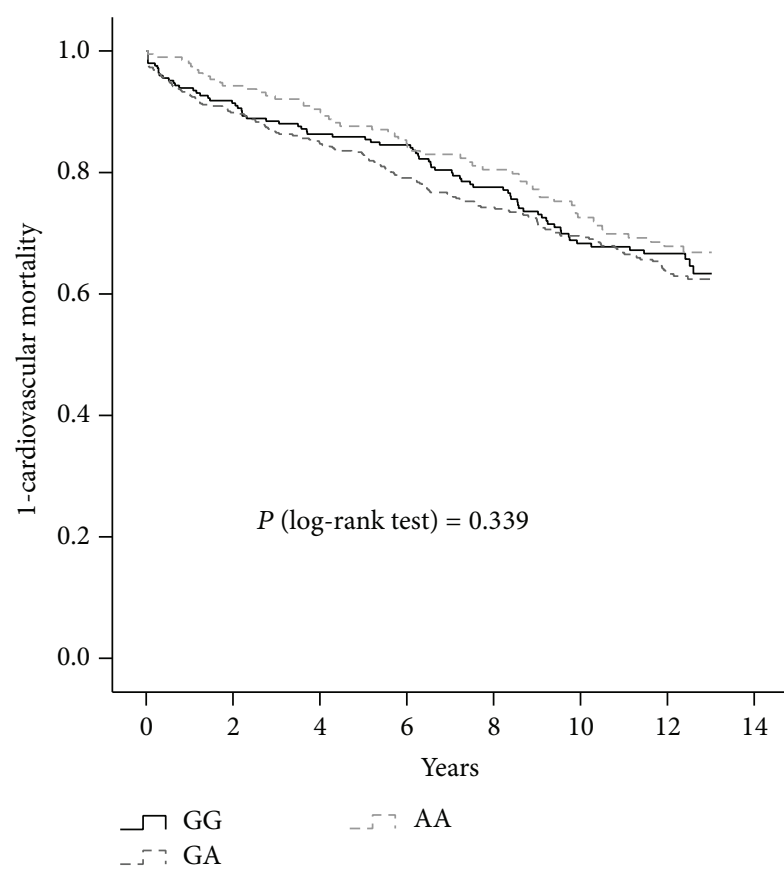

(a)

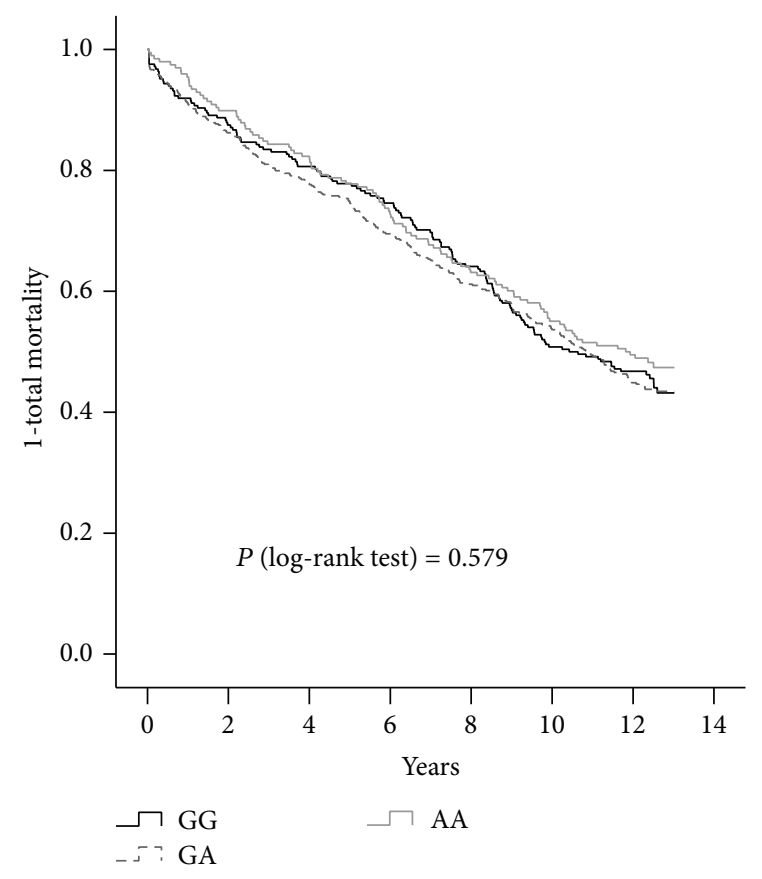

(b)

FIGURE 2: Kaplan-Meier curves showing (a) proportion without cardiovascular mortality and (b) proportion without total mortality based on different genotypes in the patients with CAD.

TABLE 2: Multivariate association between the rs4977574 genotypes and presence of coronary artery disease (CAD).

\begin{tabular}{|c|c|c|c|c|}
\hline & \multicolumn{2}{|c|}{ Dominant model $^{*}$} & \multicolumn{2}{|c|}{ Recessive model ${ }^{*}$} \\
\hline & Odds ratio (95\% CI) & $P$ & Odds ratio $(95 \% \mathrm{CI})$ & $P$ \\
\hline Crude & $1.47(1.16,1.85)$ & 0.001 & $1.35(1.07,1.72)$ & 0.013 \\
\hline Adjusted $^{1}$ & $1.47(1.16,1.86)$ & 0.002 & $1.41(1.10,1.81)$ & 0.006 \\
\hline Adjusted $^{2}$ & $1.47(1.14,1.89)$ & 0.003 & $1.36(1.05,1.77)$ & 0.018 \\
\hline
\end{tabular}

diabetes, and LDL cholesterol in the logistic regression model (Table 2).

3.2. Longitudinal Cardiovascular and Total Mortality for CAD Patients. Among the 925 patients with CAD at baseline, there were 248 with GG genotype, 479 with GA genotype, and 198 with AA genotype (Table 3). The CAD severity, based on the Gensini score, was not significantly different among all genotypes $(P=0.412)$. During the follow-up period (median of 11.0 years, interquartile range between 5.2 and 12.5 years), there was no significant difference in cardiovascular mortality or total mortality among the CAD patients with different genotypes (Figure 2). Using Cox regression analysis, the risk $\mathrm{G}$ allele was not associated with cardiovascular mortality and total mortality in either the dominant model or the recessive model after adjustment for age, gender, smoking status, past history of myocardial infarction, statins treatment, presence of hypertension, and presence of diabetes (Table 4).

\section{Discussion}

Our major findings were that the G allele of SNP rs4977574 was associated with the presence of CAD but failed to predict cardiovascular death in the Han Taiwanese patients with established CAD. The case-control findings of our study are in-line with results reported in other populations [9-13]. SNP rs4977574 is located at a locus with a gene encoding a large antisense noncoding RNA (ANRIL) [21, 22]. ANRIL might be involved in atherosclerosis by being expressed in the endothelial cells and monocyte-derived cells [21, 23]. Upstream from the locus, the cyclin-dependent kinase inhibitor $2 \mathrm{~A}(\mathrm{CDKN} 2 \mathrm{~A})$ and $2 \mathrm{~B}(\mathrm{CDKN} 2 \mathrm{~B})$ genes are associated with transcriptional regulation of ANRIL [24-26]. It is also important to note that the baseline Gensini scores were not significantly different among the CAD patients with three different genotypes. In accordance with our observations, Chen et al. [27] reported that the $9 \mathrm{p} 21$ variant is not associated with CAD severity in subjects with established CAD. Similar findings, reported in Chinese subjects with myocardial infarction, showed that severity of coronary lesion was not significantly different among different genotypes of the 9p21 variant [28]. Further investigations are warranted to clarify the underlying pathogenesis contributing to the observed association between chromosome 9p21 variants and coronary lesions.

In the present study, rs4977574 failed to predict cardiovascular or total mortality in subjects with CAD during the eleven-year period of follow-up. Our finding in CAD Chinese was different from results in subjects without CAD [15]. Furthermore, the GRACE genetic study showed the 9p21 
TABLE 3: Baseline characteristics of the subjects with coronary artery disease (CAD) according to the rs4977574 genotypes.

\begin{tabular}{|c|c|c|c|c|}
\hline & $\mathrm{GG}(N=248)$ & $\mathrm{GA}(N=479)$ & $\mathrm{AA}(N=198)$ & $P$ \\
\hline Age (year) & $66 \pm 10^{\mathrm{a}}$ & $68 \pm 10^{\mathrm{b}}$ & $68 \pm 10^{\mathrm{b}}$ & 0.009 \\
\hline Male, $n(\%)$ & $193(77.8 \%)$ & $385(80.4 \%)$ & $164(82.8 \%)$ & 0.416 \\
\hline BMI $\left(\mathrm{kg} / \mathrm{m}^{2}\right)$ & $25.7 \pm 3.4$ & $25.1 \pm 3.3$ & $25.2 \pm 3.3$ & 0.191 \\
\hline Systolic BP (mmHg) & $127 \pm 12$ & $125 \pm 13$ & $127 \pm 13$ & 0.062 \\
\hline Diastolic BP (mmHg) & $72 \pm 10$ & $71 \pm 10$ & $70 \pm 10$ & 0.264 \\
\hline Current smoking, $n(\%)$ & $75(30.2 \%)$ & $132(27.6 \%)$ & $53(26.8 \%)$ & 0.668 \\
\hline Hypertension, $n(\%)$ & $170(68.5 \%)$ & $297(62.0 \%)$ & $127(64.1 \%)$ & 0.218 \\
\hline Diabetes mellitus, $n(\%)$ & $126(50.8 \%)$ & $228(47.6 \%)$ & $92(46.5 \%)$ & 0.612 \\
\hline Previous MI & $26(10.5 \%)$ & $45(9.4 \%)$ & $21(10.6 \%)$ & 0.844 \\
\hline Statins treatment & $11(4.4 \%)$ & $35(7.3 \%)$ & $15(7.6 \%)$ & 0.275 \\
\hline Triglyceride (mmol/L) & $1.7 \pm 1.4$ & $1.5 \pm 1.0$ & $1.6 \pm 1.6$ & 0.237 \\
\hline Total cholesterol (mmol/L) & $4.7 \pm 1.2$ & $4.7 \pm 1.1$ & $4.8 \pm 1.3$ & 0.520 \\
\hline HDL cholesterol (mmol/L) & $1.1 \pm 0.3$ & $1.2 \pm 0.3$ & $1.1 \pm 0.3$ & 0.198 \\
\hline LDL cholesterol (mmol/L) & $2.8 \pm 0.9$ & $2.9 \pm 1.0$ & $3.0 \pm 1.1$ & 0.275 \\
\hline Fasting glucose (mmol/L) & $6.8 \pm 2.7$ & $6.9 \pm 2.7$ & $6.7 \pm 2.7$ & 0.780 \\
\hline Gensini score $^{*}$ & $21.5(8.0,48.8)$ & $20.0(8.0,48.0)$ & $20.0(6.4,41.3)$ & 0.412 \\
\hline
\end{tabular}

${ }^{\mathrm{a}, \mathrm{b}}$ Significant difference between a and $\mathrm{b}$.

* Data of Gensini score presented as median (interquartile range).

BMI: body mass index, BP: blood pressure, HDL: high-density lipoprotein, LDL: low-density lipoprotein, and MI: myocardial infarction.

TABLE 4: Cox regression models for the effects of rs4977574 in patients with CAD at baseline.

(a) Cardiovascular mortality

\begin{tabular}{lcccccc}
\hline & \multicolumn{3}{c}{ Dominant model } & \multicolumn{3}{c}{ Recessive model } \\
& HR & $(95 \% \mathrm{CI})$ & $P$ & HR & $(95 \% \mathrm{CI})$ & $P$ \\
\hline Age (10 years) & 1.80 & $(1.55,2.09)$ & $<0.001$ & 1.79 & $(1.54,2.08)$ & $<0.001$ \\
Male & 1.05 & $(0.78,1.41)$ & 0.741 & 1.05 & $(0.78,1.41)$ & 0.755 \\
Current smoking & 0.90 & $(0.68,1.20)$ & 0.474 & 0.91 & $(0.68,1.20)$ & 0.498 \\
Hypertension & 0.91 & $(0.71,1.160)$ & 0.456 & 0.91 & $(0.71,1.16)$ & 0.444 \\
Diabetes mellitus & 1.84 & $(1.46,2.33)$ & $<0.001$ & 1.84 & $(1.46,2.34)$ & $<0.001$ \\
Previous MI & 1.20 & $(0.83,1.72)$ & 0.336 & 1.20 & $(0.84,1.74)$ & 0.318 \\
Statins treatment & 0.75 & $(0.44,1.28)$ & 0.285 & 0.75 & $(0.44,1.28)$ & 0.289 \\
G allele & 1.25 & $(0.93,1.68)$ & 0.138 & 1.05 & $(0.80,1.37)$ & 0.729 \\
\hline
\end{tabular}

MI: myocardial infarction.

(b) Total mortality

\begin{tabular}{|c|c|c|c|c|c|c|}
\hline & \multicolumn{3}{|c|}{ Dominant model } & \multicolumn{3}{|c|}{ Recessive model } \\
\hline & HR & $(95 \% \mathrm{CI})$ & $P$ & HR & $(95 \% \mathrm{CI})$ & $P$ \\
\hline Age (10 years) & 2.12 & $(1.89,2.39)$ & $<0.001$ & 2.12 & $(1.89,2.39)$ & $<0.001$ \\
\hline Male & 1.26 & $(1.00,1.60)$ & 0.052 & 1.27 & $(1.00,1.60)$ & 0.051 \\
\hline Current smoking & 1.01 & $(0.82,1.24)$ & 0.937 & 1.01 & $(0.82,1.24)$ & 0.941 \\
\hline Hypertension & 0.91 & $(0.75,1.09)$ & 0.305 & 0.90 & $(0.75,1.08)$ & 0.271 \\
\hline Diabetes mellitus & 1.55 & $(1.30,1.84)$ & $<0.001$ & 1.54 & $(1.30,1.84)$ & $<0.001$ \\
\hline Previous MI & 1.19 & $(0.90,1.57)$ & 0.226 & 1.19 & $(0.90,1.57)$ & 0.230 \\
\hline Statins treatment & 0.76 & $(0.51,1.14)$ & 0.188 & 0.77 & $(0.51,1.15)$ & 0.202 \\
\hline G allele & 1.18 & $(0.95,1.47)$ & 0.135 & 1.11 & $(0.91,1.35)$ & 0.312 \\
\hline
\end{tabular}

MI: myocardial infarction. 
variant was significantly associated with recurrent myocardial infarction within only six months [16], but Peng et al. [28] reported that the $9 \mathrm{p} 21$ variant could not predict total mortality in subjects following myocardial infarction in a 2.5-year study. Virani et al. [17] also found no significant association between the 9p21 variant and cardiac death in subjects with established CAD in a 3.2-year follow-up. One of the main strengths of the present study was that long-term mortality in a follow-up period of 11 years was analyzed in CAD patients, using death records as the endpoint to test our hypothesis.

It is well acknowledged that the current established risk factors of atherosclerosis are not able to fully account for cardiovascular death [29]. The discrepancy probably results from different pathophysiological mechanisms. Endothelial dysfunction and monocyte activation play roles in inflammation and subsequent development of coronary lesions [30, 31]. However, the atherosclerotic biomarkers and monocyte activity were poor predictors of longitudinal cardiovascular events in patients with established CAD [32]. In fact, cardiovascular death might be associated with the critical mechanisms of plaque stabilization and arrhythmia in CAD patients [3335]. In large-scale genome-wide association studies (GWAS), susceptibility variants were determined based on case-control studies, and thus it might not be possible to prospectively predict the outcomes of diseases [36]. Previous authors have asserted that the effects of the 9p21 genetic polymorphisms might be only associated with coronary lesion but not capable of predicting cardiovascular mortality [37, 38]. Furthermore, cardiovascular death might be associated with many common diseases, which in turn may be linked to both genetic and environmental factors $[39,40]$. The association between chromosome 9p21 variants and sequential cardiovascular mortality might be abated by the medical treatment or procedural intervention during the follow-up period [41]. Along the same lines, it has been reported that the $9 \mathrm{p} 21$ variants could not predict the cardiovascular outcomes in subjects with coronary lesions after drug-eluting stent placement [42].

Cavender et al. [43] reported a total mortality of $37 \%$ in subjects with CAD at five years. In the present study, the accumulative total mortality of about $55 \%$ was observed in a median 11-year period of follow-up. Ethnic differences in mortality were reported in CAD subjects, with a higher mortality in Asians compared with that in Caucasians [44, 45]. However, the prognosis of CAD seems to be better in Chinese than that in south Asians, although the mechanism is not well understood [46]. In a recent report from a Vienna cohort study with a similar duration of follow-up [47], a lower total mortality rate (39\%) in the CAD subjects was observed, although the proportion of cardiovascular cause (57\%) was similar to that in the present study $(56.6 \%)$. Because the majority of deaths in the present study were cardiovascular causes, the total mortality findings were close to those of cardiovascular mortality.

There were some limitations in the current study. First, the cardiovascular mortality data was collected from the national mortality registry which covers $99.6 \%$ of the total population in Taiwan [48]. However, nonfatal cardiovascular events could not be traced in the follow-up assessment as these data are not provided in the database. Second, we assessed cardiovascular mortality rather than CAD mortality due to indistinct classifications of death causes; for example, some types of CAD might be classified to cardiovascular disease (ex. ICD-9 code of 429.2) [49]. Furthermore, we could not collect information on the effect of environmental factors and treatments during the follow-up period.

In conclusion, the SNP rs4977574 at chromosome 9p21 was an independent risk factor for CAD in Taiwanese individuals of Han Chinese descent. However, this SNP could not predict the total or cardiovascular mortality in these subjects, who had CAD at baseline, during the eleven-year period of follow-up.

\section{Conflict of Interests}

The authors declare that there is no conflict of interests regarding the publication of this paper.

\section{Acknowledgments}

This work was supported by Grants from Taichung Veterans General Hospital, Taichung, Taiwan, [TCVGH-1013501B and TCVGH-103DHA0500405] and National Science Council, Taiwan [NSC 102-2314-B-075A-001].

\section{References}

[1] R. Lozano, M. Naghavi, K. Foreman et al., "Global and regional mortality from 235 causes of death for 20 age groups in 1990 and 2010: a systematic analysis for the Global Burden of Disease Study 2010," The Lancet, vol. 380, no. 9859, pp. 2095-2128, 2013.

[2] S. Zdravkovic, A. Wienke, N. L. Pedersen, M. E. Marenberg, A. I. Yashin, and U. De Faire, "Heritability of death from coronary heart disease: a 36-year follow-up of 20966 Swedish twins," Journal of Internal Medicine, vol. 252, no. 3, pp. 247-254, 2002.

[3] L. Zhou, X. Zhang, M. He et al., "Associations between single nucleotide polymorphisms on chromosome 9p21 and risk of coronary heart disease in Chinese Han population," Arteriosclerosis, Thrombosis, and Vascular Biology, vol. 28, no. 11, pp. 20852089, 2008.

[4] Q. Zhang, X.-F. Wang, S.-S. Cheng et al., "Three SNPs on chromosome 9p21 confer increased risk of myocardial infarction in Chinese subjects," Atherosclerosis, vol. 207, no. 1, pp. 26-28, 2009.

[5] J. Guo, W. Li, Z. Wu, X. Cheng, Y. Wang, and T. Chen, "Association between 9p21. 3 genomic markers and coronary artery disease in East Asians: a meta-analysis involving 9, 813 cases and 10, 710 controls," Molecular Biology Reports, vol. 40, no. 1, pp. 337-343, 2013.

[6] Y. Gong, A. L. Beitelshees, R. M. Cooper-DeHoff et al., "Chromosome 9p21 haplotypes and prognosis in white and black patients with coronary artery disease," Circulation: Cardiovascular Genetics, vol. 4, no. 2, pp. 169-178, 2011.

[7] T. L. Assimes, J. W. Knowles, A. Basu et al., "Susceptibility locus for clinical and subclinical coronary artery disease at chromosome 9p21 in the multi-ethnic ADVANCE study," Human Molecular Genetics, vol. 17, no. 15, pp. 2320-2328, 2008. 
[8] R. McPherson, A. Pertsemlidis, N. Kavaslar et al., "A common allele on chromosome 9 associated with coronary heart disease," Science, vol. 316, no. 5830, pp. 1488-1491, 2007.

[9] S. Kathiresan, B. F. Voight, S. Purcell et al., "Genome-wide association of early-onset myocardial infarction with single nucleotide polymorphisms and copy number variants," Nature Genetics, vol. 41, no. 3, pp. 334-341, 2009.

[10] L. Qi, J. Ma, Q. Qi, J. Hartiala, H. Allayee, and H. Campos, "Genetic risk score and risk of myocardial infarction in hispanics," Circulation, vol. 123, no. 4, pp. 374-380, 2011.

[11] T. M. Beckie, M. W. Groër, and J. W. Beckstead, "The relationship between polymorphisms on chromosome 9p21 and age of onset of coronary heart disease in black and white women," Genetic Testing and Molecular Biomarkers, vol. 15, no. 6, pp. 435442, 2011.

[12] S. Saade, J.-B. Cazier, M. Ghassibe-Sabbagh et al., "Large scale association analysis identifies three susceptibility loci for coronary artery disease," PLoS ONE, vol. 6, no. 12, Article ID e29427, 2011.

[13] J. Y. Lee, B. S. Lee, D. J. Shin et al., "A genome-wide association study of a coronary artery disease risk variant," Journal of Human Genetics, vol. 58, no. 3, pp. 120-126, 2013.

[14] L. Qi, J. M. Li, H. Sun et al., "Association between gene polymorphisms and myocardial infarction in Han Chinese of Yunnan province," Chinese Journal of Medical Genetics, vol. 29, no. 4, pp. 413-419, 2012.

[15] K. Gransbo, P. Almgren, M. Sjogren et al., "Chromosome 9p21 genetic variation explains $13 \%$ of cardiovascular disease incidence but does not improve risk prediction," Journal of Internal Medicine, vol. 274, no. 3, pp. 233-240, 2013.

[16] I. Buysschaert, K. F. Carruthers, D. R. Dunbar et al., "A variant at chromosome 9 p21 is associated with recurrent myocardial infarction and cardiac death after acute coronary syndrome: the GRACE genetics study," European Heart Journal, vol. 31, no. 9, pp. 1132-1141, 2010.

[17] S. S. Virani, A. Brautbar, V.-V. Lee et al., "Chromosome 9p21 single nucleotide polymorphisms are not associated with recurrent myocardial infarction in patients with established coronary artery disease," Circulation Journal, vol. 76, no. 4, pp. 950-956, 2012.

[18] W. T. Friedewald, R. I. Levy, and D. S. Fredrickson, "Estimation of the concentration of low-density lipoprotein cholesterol in plasma, without use of the preparative ultracentrifuge," Clinical Chemistry, vol. 18, no. 6, pp. 499-502, 1972.

[19] G. G. Gensini, "A more meaningful scoring system for determining the severity of coronary heart disease," The American Journal of Cardiology, vol. 51, no. 3, p. 606, 1983.

[20] L. N. Tseng, Y. H. Tseng, Y. D. Jiang et al., "Prevalence of hypertension and dyslipidemia and their associations with micro- and macrovascular diseases in patients with diabetes in Taiwan: an analysis of nationwide data for 2000-2009," Journal of the Formosan Medical Association, vol. 111, no. 11, pp. 625-636, 2012.

[21] H. M. Broadbent, J. F. Peden, S. Lorkowski et al., "Susceptibility to coronary artery disease and diabetes is encoded by distinct, tightly linked SNPs in the ANRIL locus on chromosome 9p," Human Molecular Genetics, vol. 17, no. 6, pp. 806-814, 2008.

[22] K. Silander, H. Tang, S. Myles et al., "Worldwide patterns of haplotype diversity at $9 \mathrm{p} 21.3$, a locus associated with type 2 diabetes and coronary heart disease," Genome Medicine, vol. 1, no. 5, article 51, 2009.
[23] L. M. Holdt, F. Beutner, M. Scholz et al., "ANRIL expression is associated with atherosclerosis risk at chromosome 9p21," Arteriosclerosis, Thrombosis, and Vascular Biology, vol. 30, no. 3, pp. 620-627, 2010.

[24] A. Motterle, X. Pu, H. Wood et al., "Functional analyses of coronary artery disease associated variation on chromosome 9p21 in vascular smooth muscle cells," Human Molecular Genetics, vol. 21, no. 18, pp. 4021-4029, 2012.

[25] M. S. Cunnington, M. S. Koref, B. M. Mayosi, J. Burn, and B. Keavney, "Chromosome 9p21 SNPs associated with multiple disease phenotypes correlate with ANRIL expression," PLoS Genetics, vol. 6, no. 4, Article ID e1000899, 2010.

[26] C.-L. Kuo, A. J. Murphy, S. Sayers et al., "Cdkn2a is an atherosclerosis modifier locus that regulates monocyte/macrophage proliferation," Arteriosclerosis, Thrombosis, and Vascular Biology, vol. 31, no. 11, pp. 2483-2492, 2011.

[27] S. N. Chen, C. M. Ballantyne, A. M. Gotto Jr., and A. J. Marian, "The 9p21 susceptibility locus for coronary artery disease and the severity of coronary atherosclerosis," BMC Cardiovascular Disorders, vol. 9, article 3, 2009.

[28] W. H. Peng, L. Lu, Q. Zhang et al., "Chromosome 9p21 polymorphism is associated with myocardial infarction but not with clinical outcome in Han Chinese," Clinical Chemistry and Laboratory Medicine, vol. 47, no. 8, pp. 917-922, 2009.

[29] J. G. Canto, C. I. Kiefe, W. J. Rogers et al., "Atherosclerotic risk factors and their association with hospital mortality among patients with first myocardial infarction (from the National Registry of Myocardial Infarction)," The American Journal of Cardiology, vol. 110, no. 9, pp. 1256-1261, 2012.

[30] M. K. Reriani, A. J. Flammer, A. Jama, L. O. Lerman, and A. Lerman, "Novel functional risk factors for the prediction of cardiovascular events in vulnerable patients following acute coronary syndrome," Circulation Journal, vol. 76, no. 4, pp. 778783, 2012

[31] K.-W. Liang, W.-J. Lee, W.-L. Lee, C.-T. Ting, and W. H.-H. Sheu, "Decreased ratio of high-molecular-weight to total adiponectin is associated with angiographic coronary atherosclerosis severity but not restenosis," Clinica Chimica Acta, vol. 405, no. 1-2, pp. 114-118, 2009.

[32] B. J. Arsenault, P. Barter, D. A. Demicco et al., "Prediction of cardiovascular events in statin-treated stable coronary patients by lipid and nonlipid biomarkers," Journal of the American College of Cardiology, vol. 57, no. 1, pp. 63-69, 2010.

[33] A. Baruch, N. van Bruggen, J. B. Kim, and J. E. Lehrer-Graiwer, "Anti-inflammatory strategies for plaque stabilization after acute coronary syndromes," Current Atherosclerosis Reports, vol. 15, no. 6, p. 327, 2013.

[34] L. Y. Chen, N. Sotoodehnia, P. Buzkova et al., "Atrial fibrillation and the risk of sudden cardiac death: the atherosclerosis risk in communities study and cardiovascular health study," JAMA Internal Medicine, vol. 173, no. 1, pp. 29-35, 2013.

[35] P. Tung and C. M. Albert, "Causes and prevention of sudden cardiac death in the elderly," Nature Reviews Cardiology, vol. 10, no. 3, pp. 135-142, 2013.

[36] A. C. J. W. Janssens and C. M. van Duijn, "Genome-based prediction of common diseases: advances and prospects," Human Molecular Genetics, vol. 17, no. 2, pp. R166-R173, 2008.

[37] D. Ardissino, C. Berzuini, P. A. Merlini et al., "Influence of 9p21.3 genetic variants on clinical and angiographic outcomes in early-onset myocardial infarction," Journal of the American College of Cardiology, vol. 58, no. 4, pp. 426-434, 2011. 
[38] J. B. Muhlestein and J. L. Anderson, “The 9p21.3 genetic region and coronary heart disease where do we go from here?" Journal of the American College of Cardiology, vol. 58, no. 4, pp. 435-437, 2011.

[39] A. J. Marian, “The enigma of genetics etiology of atherosclerosis in the post-GWAS era," Current Atherosclerosis Reports, vol. 14, no. 4, pp. 295-299, 2012.

[40] M. I. McCarthy, G. R. Abecasis, L. R. Cardon et al., "Genomewide association studies for complex traits: consensus, uncertainty and challenges," Nature Reviews Genetics, vol. 9, no. 5, pp. 356-369, 2008.

[41] R. S. Patel, S. Su, I. J. Neeland et al., “The chromosome 9p21 risk locus is associated with angiographic severity and progression of coronary artery disease," European Heart Journal, vol. 31, no. 24, pp. 3017-3023, 2010.

[42] P. Hoppmann, A. Erl, S. Türk et al., "No association of chromosome 9p21.3 variation with clinical and angiographic outcomes after placement of drug-eluting stents," JACC: Cardiovascular Interventions, vol. 2, no. 11, pp. 1149-1155, 2009.

[43] M. A. Cavender, K. P. Alexander, S. Broderick et al., "Long-term morbidity and mortality among medically managed patients with angina and multivessel coronary artery disease," American Heart Journal, vol. 158, no. 6, pp. 933-940, 2009.

[44] P. Wilkinson, J. Sayer, K. Laji et al., "Comparison of case fatality in south Asian and white patients after acute myocardial infarction: observational study," British Medical Journal, vol. 312, no. 7042, pp. 1330-1333, 1996.

[45] M. J. Zaman and R. S. Bhopal, "New answers to three questions on the epidemic of coronary mortality in south Asians: incidence or case fatality? Biology or environment? Will the next generation be affected?” Heart, vol. 99, no. 3, pp. 154-158, 2013.

[46] A. S. Koh, L. W. Khin, L. M. Choi et al., "Percutaneous coronary intervention in asians- are there differences in clinical outcome?" BMC Cardiovascular Disorders, vol. 11, article 22, 2011.

[47] G. Goliasch, M. E. Kleber, B. Richter et al., "Routinely available biomarkers improve prediction of long-term mortality in stable coronary artery disease: the Vienna and Ludwigshafen Coronary Artery Disease (VILCAD) risk score," European Heart Journal, vol. 33, no. 18, pp. 2282-2289, 2012.

[48] C. P. Wen, S. P. Tsai, and W.-S. I. Chung, "A 10-year experience with universal health insurance in Taiwan: measuring changes in health and health disparity," Annals of Internal Medicine, vol. 148, no. 4, pp. 258-267, 2008.

[49] S. De Henauw, P. De Smet, W. Aelvoet, M. Kornitzer, and G. De Backer, "Misclassification of coronary heart disease in mortality statistics. Evidence from the WHO-MONICA Ghent-Charleroi study in Belgium," Journal of Epidemiology and Community Health, vol. 52, no. 8, pp. 513-519, 1998. 


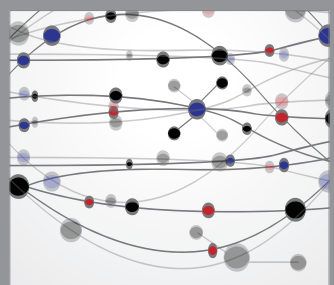

The Scientific World Journal
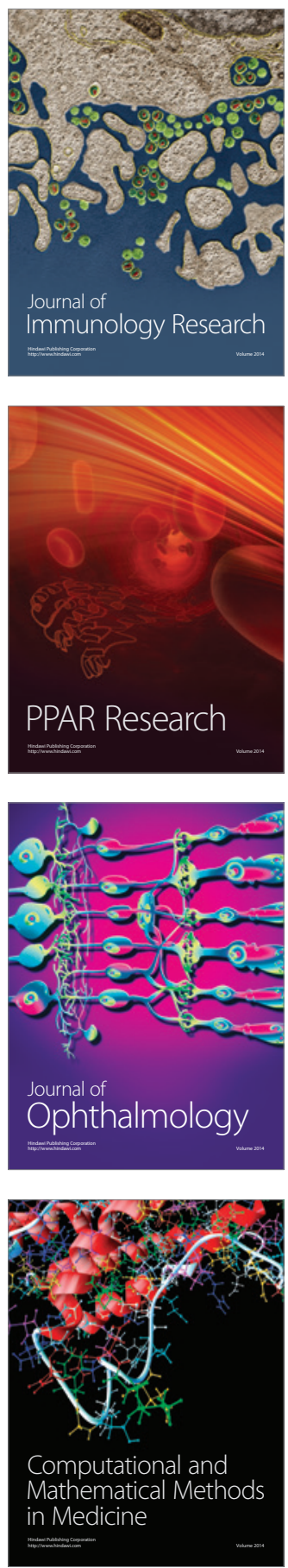

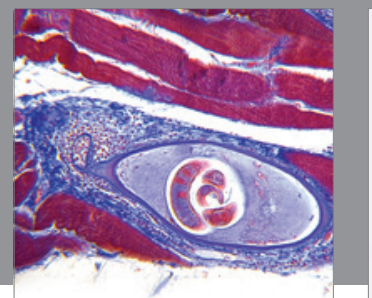

Gastroenterology

Research and Practice
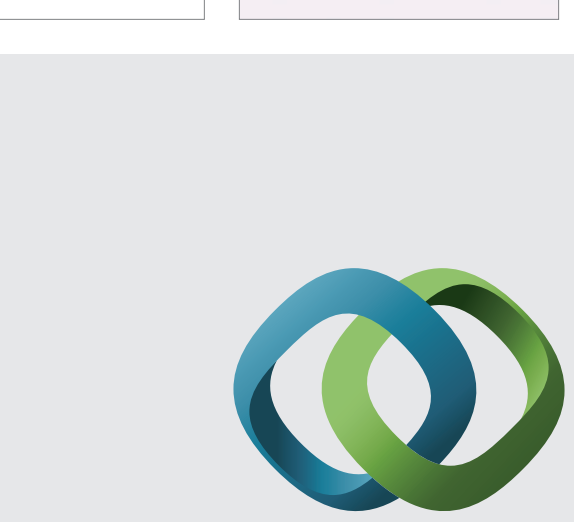

\section{Hindawi}

Submit your manuscripts at

http://www.hindawi.com
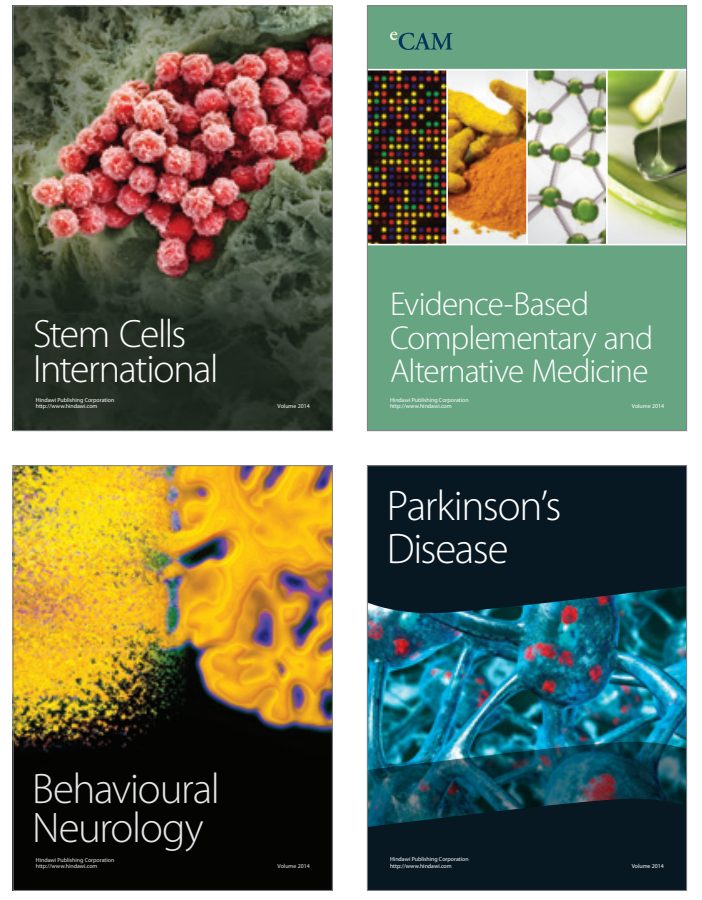
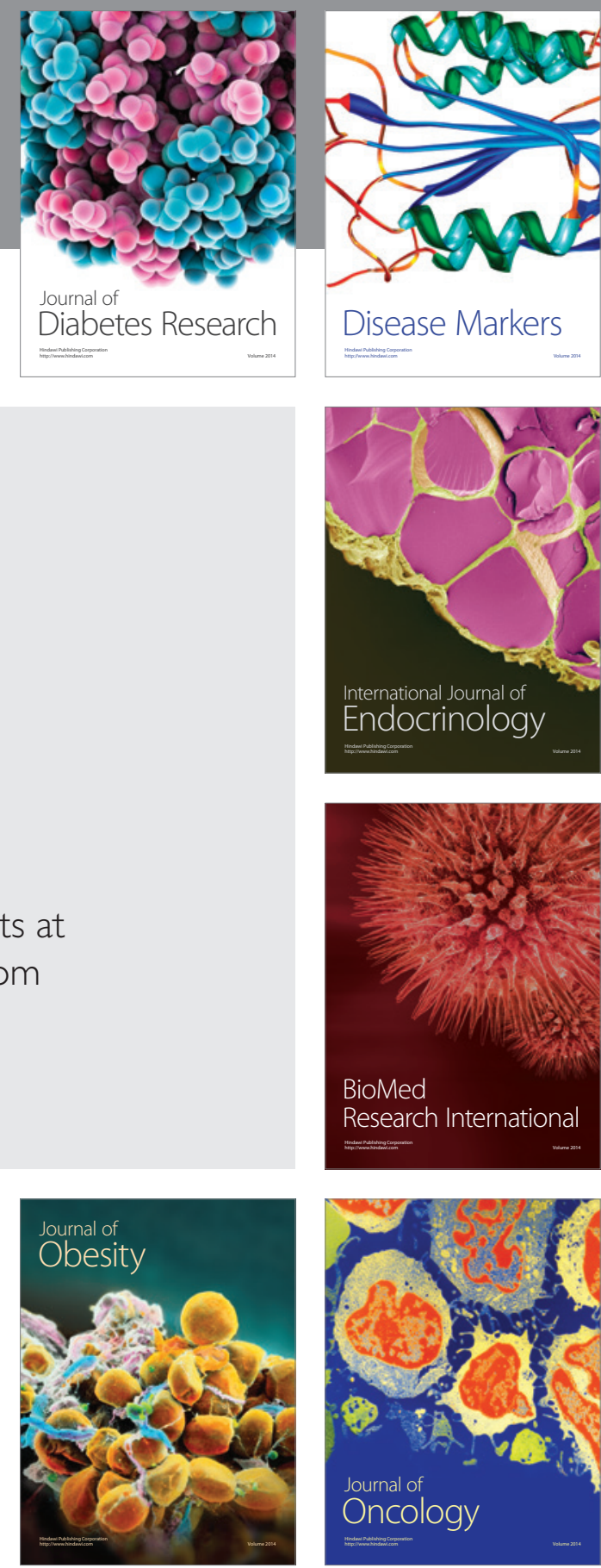

Disease Markers
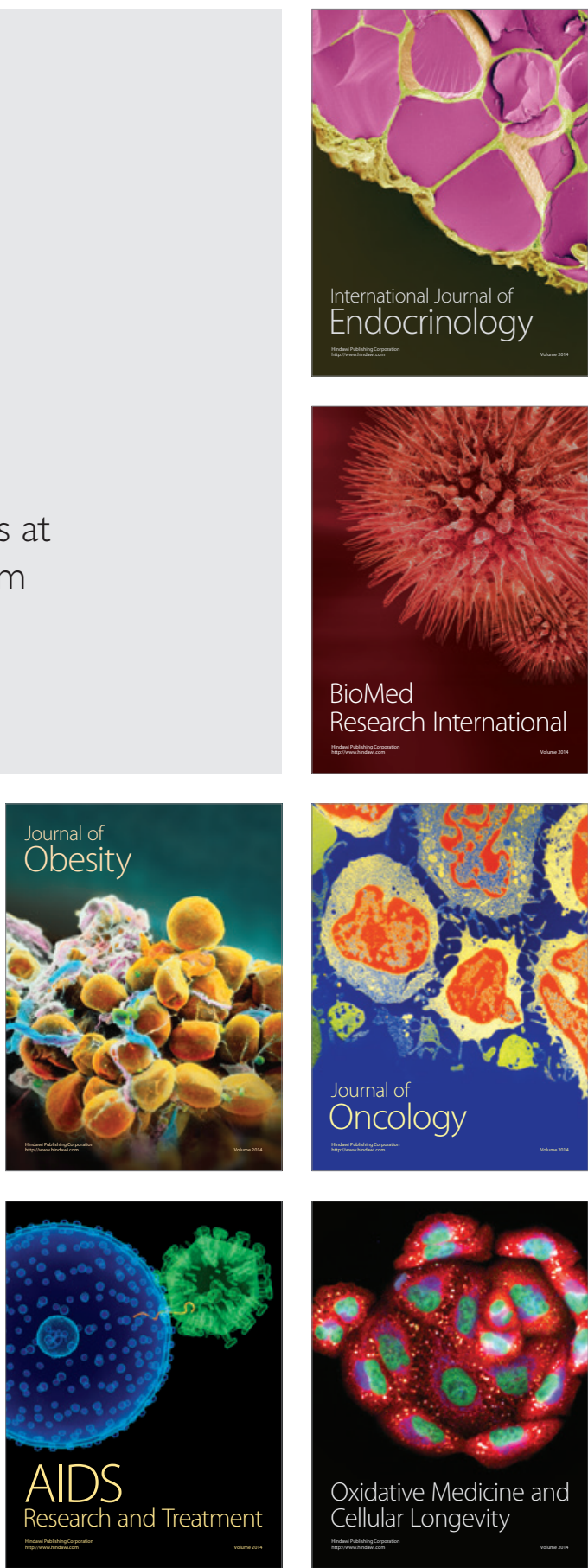